\title{
Future Opportunities in DIS
}

\author{
Joël Feltesse * \\ CEA, DSM/DAPNIA \\ CE-Saclay, Gif-sur-Yvette, France
}

\begin{abstract}
The talk presents a personal view on future prospects in DIS. The open questions which have not been fully answered in lepton-nucleon Deep Inelastic Scattering by the past and present facilities are sketched. The proposals of future facilities are briefly reviewed and discussed.
\end{abstract}

\section{Introduction}

The year 2007 is a turning point of high energy physics. Data taking at HERA in the e-p collider experiments H1 and ZEUS as well as in the fixed target experiment HERMES should come to an end on 30th June. The new proton-proton collider LHC should start to be commissioned at CERN by the end of 2007. It is timely to ask ourselves whether this new era is the end of Deep Inelastic lepton-nucleon Physics (DIS), a bit more than 50 years after the pioneering work of R. Hoftater at SLAC [2] on elastic electron scattering off hydrogen, deuterium and helium. This talk gives a personal view on the perspective in DIS rather than a summary of the very rich parallel session on Future of DIS. Section 2 presents what I see as the most important issues in DIS in 2007. Section 3 gives an overview of the new DIS projects which have been proposed or are under consideration. Section 4 reminds how complementary are the e-p, p-p,e-A, A-A facilities to get more insight into DIS physics. Finally a tentative conclusion is given in section 5 .

\section{Open questions}

After decades of effort in fixed target and collider experiments the momentum distribution of quark and gluon in the proton is known at a fair level of accuracy which is further commented below. However even in the kinematic domain reached so far there are still a lot to explore.

- How does the proton's spin $1 / 2$ originate from the dynamics of quarks and gluons? In the domain where a quark carries almost the whole momentum of the proton $(x \rightarrow 1)$, what is the $d / u$ ratio?

- How well do we know the quark and gluon distributions in nuleons imbedded in nuclei?

- As the LHC is about to start running we should ask ourselves if the Parton Distribution Functions (PDF), mainly extracted from deep inelastic scattering experiments, have the necessary precision for predicting cross sections at a $14 \mathrm{TeV}$ pp collider.

- HERA has open up the small $x$ and the hard diffraction domains. Are these domains well understood within QCD?

- At last, but not the least, is it hopeless to find a new interaction between the quark and lepton sectors beyond the standard electroweak interaction (and the gravitation)?

A few examples to illustrate these important questions follow.

*Also at DESY and University Hamburg, Helmoltz Humboldt Research Award. 


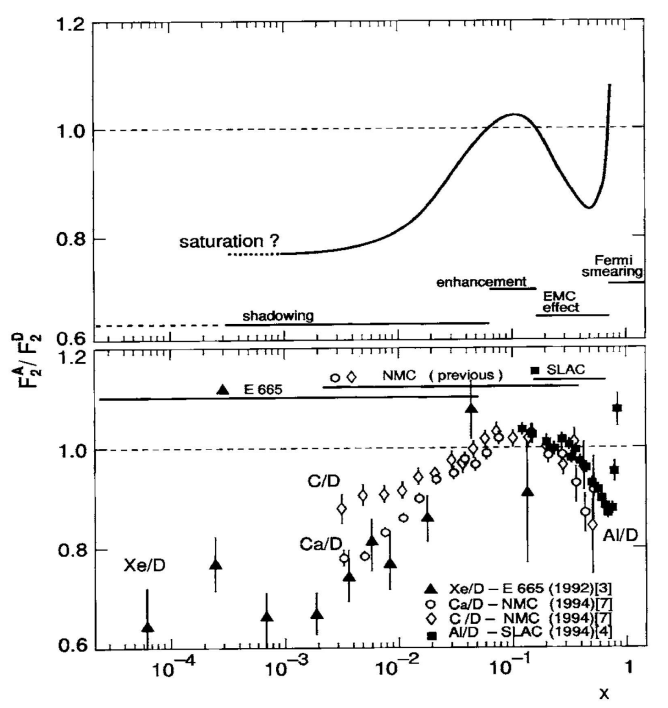

Figure 1: Upper: an idealized depiction of the ratio of the structure function of a nucleus $F_{2}^{A}\left(x, Q^{2}\right)$ per nucleon to $F_{2}^{D}\left(x, Q^{2}\right)$ of Deuterium from [5]. Lower: measured structure functions relative to Deuterium from [6].

\subsection{Proton structure}

The proton spin sum-rule :

$$
\frac{1}{2}=\Delta \Sigma+\Delta G+L_{q}+L_{g}
$$

states that the proton spin is the sum of the quark $(\Delta \Sigma)$ and gluon intrinsic spins $(\Delta G)$ and orbital angular momentum $\left(L_{q}, L_{g}\right.$ contributions). If the quarks $\Delta \Sigma$ term is at present rather well known $\Delta \Sigma \approx 0.25$ the other terms are practically unknown. Precise masurements of scaling violation and measuement of photon-gluon processes with polarised beams and polarised targets would give access to $\Delta G$. The only hope to have some hint on the orbital angular momentum would be through the JI Sum rule [3]:

$$
J^{q}=\frac{1}{2} \int_{-1}^{+1} x d x\left[H^{q}(x, \xi, t \rightarrow 0) E^{q}(x, \xi, t \rightarrow 0)\right]=\frac{1}{2} \Delta \Sigma+L_{q}
$$

where $H^{q}$ and $E^{q}$ are two General Parton Distributions (GPD) which are related to the correlation between the momentum and the spatial distributions of partons in the nucleon. Measurement of GPDs is a new field which has started recently. A model independent extraction of the GPDs is out of reach of the present and proposed future facilities. However precise measurements in the accessible range should bring some insight into this fully unknown domain of the structure of the proton.

\subsection{Quarks and gluons in Nuclei}

After the pioneering experiments at SLAC, the experiments of DIS off proton and neutron have been extended to scattering of muons off heavy nuclei at CERN. In 1982, it came as a 


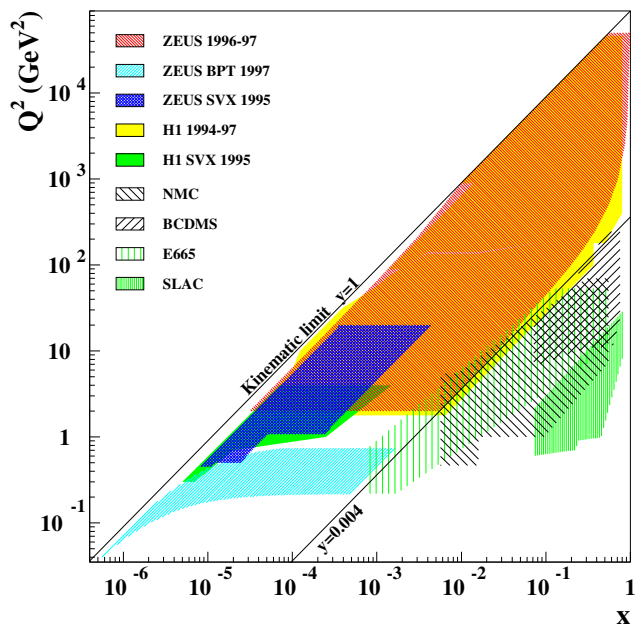

Figure 2: Kinematic regions in $x$ and $Q^{2}$ covered by fixed target experiments and the H1 and ZEUS experiments at HERA.

surprise when the EMC experiment observed a nuclear dependence to the nuclear structure $F_{2}\left(x, Q^{2}\right)$ in iron relative to that for Deuterium : a rise at $x \sim 0.05$ and a strong drop at $x \sim 0.5$ [4]. Several dedicated fixed target experiments [6] confirm the effect at large $x$ and extended the measurement down to about $x \sim 10^{-3}$ where a strong drop, called the nuclear shadowing effect, was observed ( Figure 1). It is striking that about the same time a group of theoreticians from Saint-Petersburg predicted that the gluon distribution in the proton should rise with decreasing $x$ and eventually saturate at a dynamical $Q_{s}^{2}$ value [7]. Simple considerations predict a significant dependence of the saturation scale on the atomic number A [8]:

$$
Q_{s}^{2} \sim\left(\frac{A}{x}\right)^{\frac{1}{3}}
$$

The nucleus is expected to be an amplifier of the saturation scale. It is tempting to connect these two phenomena : shadowing i.e. $x g_{A}\left(x, Q^{2}\right)<x g\left(x, Q^{2}\right)$ and saturation i.e. $\left.x g\left(x, Q^{2}\right)<x g_{\text {sat }}\left(x, Q^{2}\right)\right)$, although it is not yet proved that saturation can explain the shadowing.

At HERA, the gluonic structure of the proton has been measured down to $x \sim 10^{-4}$. In contrast, the gluon in nuclei is completely unknown. Extrapolation of the gluon distribution in $\mathrm{Pb}$ nuclei to $x$ values $\sim 10^{-3}$ differ by a factor of three pending on the model of shadowing [9]. The measurement of the gluon density in nuclei at this very low $x$ values may turn out to be vital [10] to understand the formation and the thermalization of the strongly interacting Quark Gluon Pasma (QGP) at RHIC and LHC.

\subsection{Understanding and Exploring QCD}

The H1 and ZEUS at HERA discovered surprising behaviour in previously unexplored regions. Principle among these was the discovery that the proton contains a substantial 


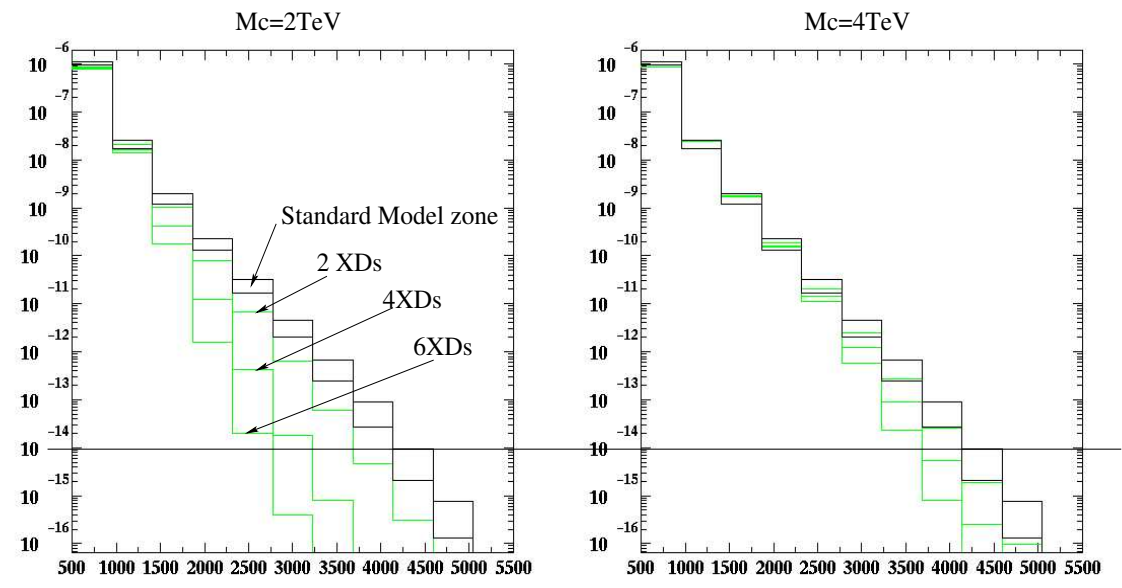

Figure 3: Di-jets cross section $(\mathrm{mb})$ predictions at LHC versus $P_{t}(\mathrm{GeV})$ with two compactification scales. At $M_{c}=4 \mathrm{TeV}$ (right) the predictions fall into the Standard Model band bounded by the present uncertainties on the PDFs [15]. The horizontal line corresponds to an integrated luminosity of $100 \mathrm{fb}^{-1}$.

quantity of quarks and gluons at very low $x$ values. There is a steep rise of the structure function $F_{2}$ and of the gluon density with $x$ decreasing [11]. Although this feature had been anticipated qualitatively, the distribution of quark and gluon momenta at low $x$ had not been predicted and its quantitative understanding represents still today a significant theoretical challenge which needs to be guided by data at lower $x$ values. Also, the possibility that the gluon component might saturate remains an unanswered question. The inclusive HERA data can be fully described by the DGLAP equations [12] and there is no evidence that the saturation domain has been reached. The $\left(x, Q^{2}\right)$ position of the saturation limit requires further experimental guidance. In addition, diffractive processes contributing to deep inelastic processes were found to be more substantial than expected [13]; another feature which still lacks a theoretical understanding and requires further experimental explorations.

\subsection{Precision of PDFs}

The study of the structure of the proton is not only a fascinating topic in its own right but also a perequisit to predict the cross sections of production of the Higgs Boson or other Standard Model phenomena at the LHC. As has been shown at this conference [14] there is still an uncertainty of about $10 \%$ on the cross sections of $\mathrm{W}$ bosons at LHC, pending on the data set which are used to extract the gluon density and the method to take into account the heavy quark mass threshold. The uncertainty on PDFs could be a severe limitation to the discovery of new phenomena at LHC. A typical example is the discovery of extra-dimensions which would be restricted to a compactification scale of $2 \mathrm{TeV}$ [15] ( Figure 3). 


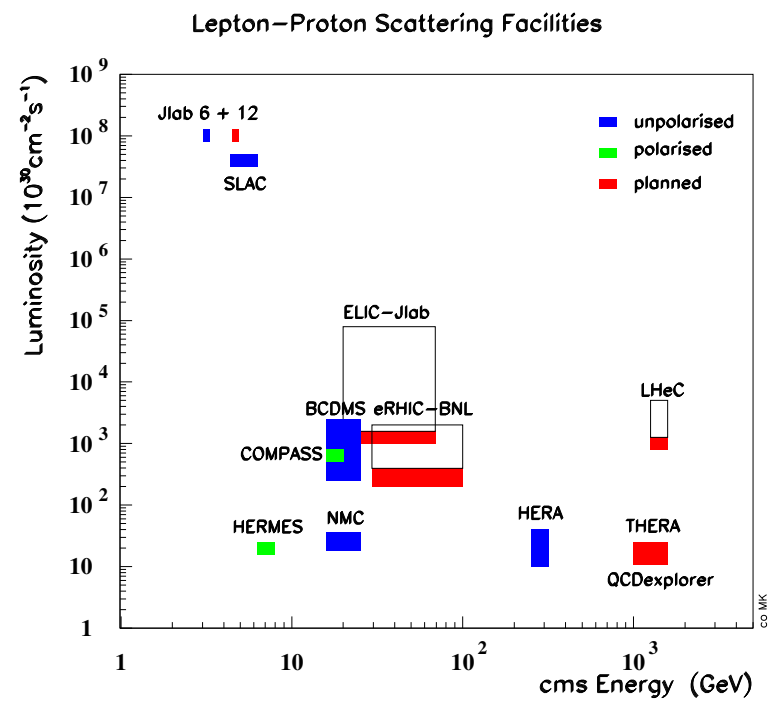

Figure 4: Energy and luminosity for existing (dark, blue boxes) and prospects for new (grey, red boxes) facilities in lepton-proton DIS. For the polarised facilities (green boxes) no dilution factor has been taken into account.

\subsection{Relation between quark and lepton sector}

Despite intensive searches with the full HERA statistics of $1 \mathrm{fb}^{-1}\left(0.5 \mathrm{fb}^{-1}\right.$ per experiment) no clear evidence for new physics has been found. The hope to find a relation between the quark and the lepton sectors beyond the standard Electroweak interaction has not been fulfilled. But the new physics which is expected at the TeV level may generate new motivations for futher study of the electron parton interaction.

\section{Proposed projects}

\subsection{JLAB $12 \mathrm{GeV}$}

Amongst the future facilities which are under consideration to extend the study of the lepton nucleon deep inelastic scattering, the most advanced is the $12 \mathrm{GeV}$ Upgrade of the electron beam at Jefferson Lab ( lower part of Figure 7). Scope of the project includes doubling the accelerator beam energy by adding new cryomodules and upgrading magnets, a new experimental Hall and upgrades to the existing three experimental Halls [16]. The preliminary baseline range has been approved in February 2006, Critical Decision 1 by the DOE (CD-1). The construction and performance baseline (CD-2) is expected to be approved in September 2007. The construction could start in 2008 and the commissioning of the accelerator by 2013. Although the $5 \mathrm{GeV}$ energy in the center-of-mass is rather modest, the huge luminosity, up to $10^{7}$ higher than at HERMES when no dilution nor average polarisation factor are applied ( Figure 4), gives access to the valence quark region in the perturbative QCD domain up to $Q^{2}=8 \mathrm{GeV}^{2}$. It is expected to allow for a precise measurement of the spin and flavour dependence of the valence quark region, both in nucleons 


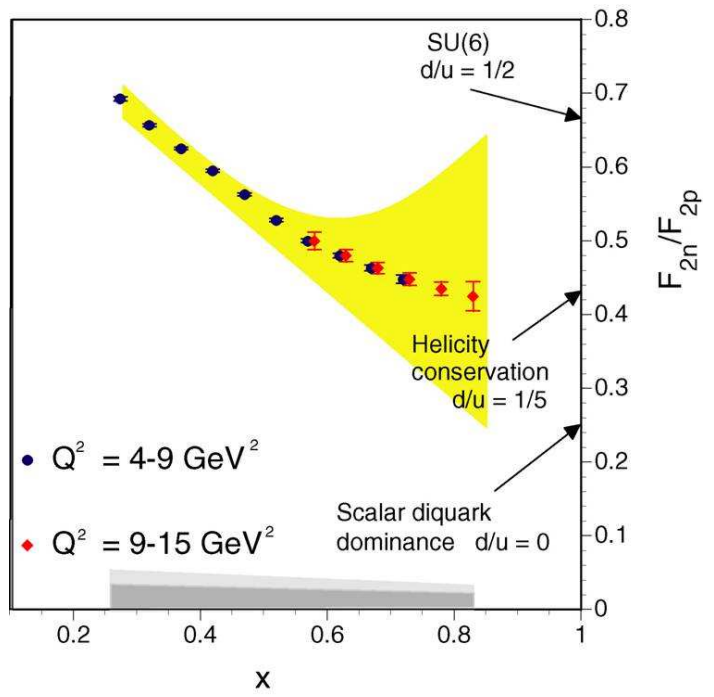

Figure 5: Predicted precision on $F_{2}^{n} / F_{2}^{p}$ at JLab $12 \mathrm{GeV}$ compared to the present uncertainty ( yellow band) and to various theoretical predictions as $x \rightarrow 1$.

and nuclei. It gives access to the unique domain where the proton momentum is carried by one parton. A beautiful example would be to solve a long standing issue : the $d / u$ ratio as $x \rightarrow 1$ ( Figure 5).

\section{$3.2 \quad$ EIC}

A new high energy electron-ion collider (EIC) has been identified [17] as an optimal experimental approach to address essential questions on quark gluon structure of the proton and of the nuclei and to explore QCD dynamics at high density of quark-gluon matter. The goals for the electron-ion collider include:

- A center-of-mass range from $20 \mathrm{GeV}$ (to overlap with muon fixed target experiments at $\mathrm{CERN}$ ) to $100 \mathrm{GeV}$ to access the low $x$ domain and explore saturation phenomena in nuclei.

- A high luminosity electron/positron ion collider of at least $10^{33} / \mathrm{cm}^{2} / \mathrm{s}$.

- Polarized ( 70\%) electron, positron, proton and neutron effective beams.

- Nuclear beams from Deuterium to Uranium.

There are at present two concepts to realize EIC:

1. To construct an electron beam (either ring or linac) to collide with the existing RHIC ion complex. This is known as eRHIC

2. To construct an ion complex to collide with upgraded CEBAF accelerator. This is known as Electron-Light-Ion-Collider or ELIC. 


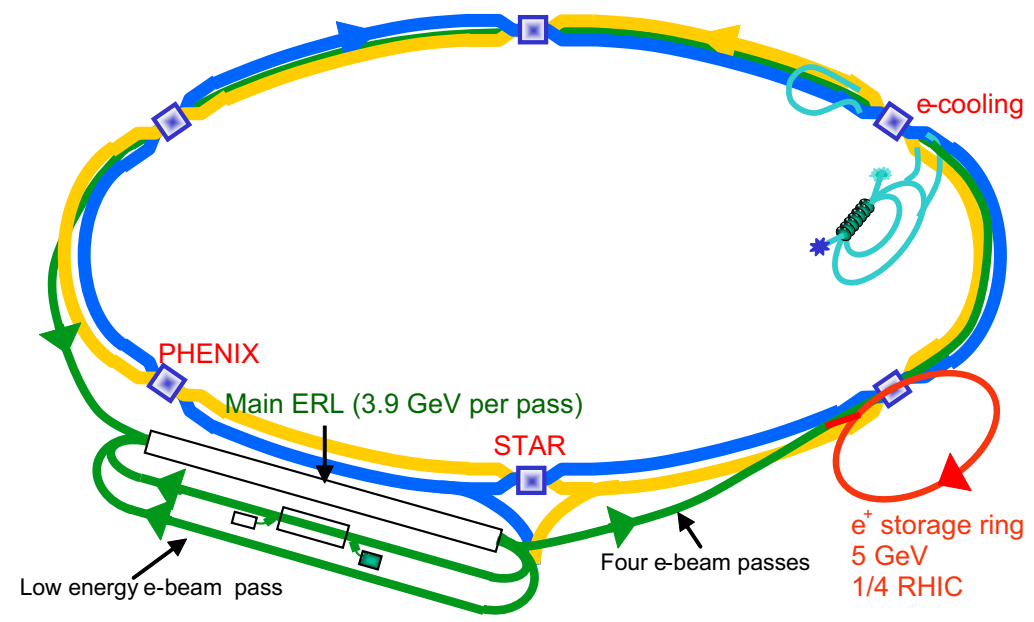

Figure 6: Design layout of the eRHIC collider based on the Energy Recovery Linac.

\section{$3.2 .1 e R H I C$}

The existing RHIC complex allows polarised protons to be stored for collisions from 30 to $250 \mathrm{GeV} / \mathrm{c}$. It should be soon (by 2010) possible to accelerate all nuclei up to Uranium to $100 \mathrm{GeV} / \mathrm{c}$ per nucleon (approved project EBIS) and to have a 10-fold increase of luminosity. This is the project RHIC-II which is not yet approved but expected to be ready by 2014 . On longer term there are still two design options to realize eRHIC:

1. An eRHIC Linac-ring design, which involves construction of a $10 \mathrm{GeV} / \mathrm{c}$ Energy Recovery Linac (ERL)( Figure 6). It is presently the most promising design [18]. As many as four electron-ion interaction points are possible. The peak luminosity is $2.610^{33} / \mathrm{cm}^{2} / \mathrm{s}$ with potential for upgrade (Figure 4). The high intensity polarized electron current source and the energy recovery capability require extensive R\&D effort.

2. An eRHIC ring-ring (RR) design, which involves construction of a ring of $10 \mathrm{GeV} / \mathrm{c}$ electrons or positrons along side the RHIC. The storage ring design is more mature than the ERL-based design. It is based on existing technology but the luminosity would be 5 to 10 times smaller than in the ERL option. Today, both designs have similar cost and could be operational at BNL by 2019 [18].

\subsubsection{ELIC}

An ambitious design is pursued at Jefferson Lab [16]. It uses the $12 \mathrm{GeV}$ upgraded CEBAF linear accelerator and requires the construction of a 30 to $225 \mathrm{GeV}$ ion storage ring in its vicinity (Figure 7). A peak luminosity of up to about $10^{35} / \mathrm{cm}^{2} / \mathrm{s}$ looks achievable for electron-light ion collisions at a center-of-mass energy between 20 and $90 \mathrm{GeV}$ (Figure 4). It requires a vigorous $\mathrm{R} \& \mathrm{D}$ but could start at $10^{33} / \mathrm{cm}^{2} / \mathrm{s}$ with state-of-the art technology except for electron cooling. Four interaction regions for detectors are possible. It could be operational by 2024 . 


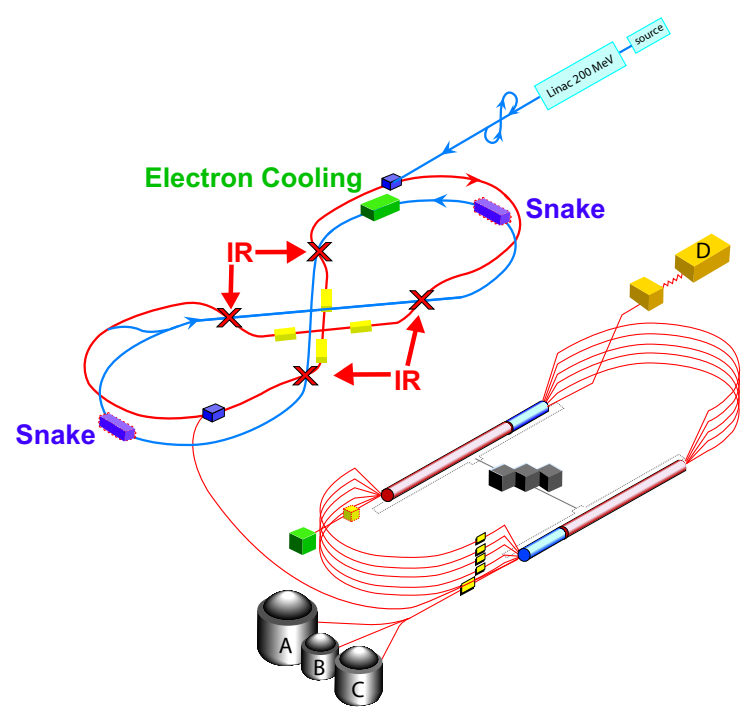

Figure 7: ELIC lay out. Upper part : ion complex. Lower part : the $12 \mathrm{geV}$ upgrade of CEBAF.

\subsubsection{Highlights of EIC Physics}

The scientific case for EIC addresses questions central to the study and the exploration of QCD. Here a very small selection of the highlights is given as an illustration of the rich physics programme of EIC.

The spin structure of the nucleon A very spectacular improvement on the precision of the contribution of gluons to the spin of the proton is expected [19] from measurement of scaling violations of the $g_{1}\left(x, Q^{2}\right)$ spin structure functions down to $x \sim 10^{-4}$ or (and) of the measurement of the photon gluon process from charm production (Figure 8). The huge luminosity gives also a reasonable hope that it would be possible to quantify at some level how the orbital motion of quarks in the nucleon contributes to the nucleon spin from the measurement of the General Parton Distributions in hard exclusive processes [17].

Nuclear matter The low $x$ domain $(x<0.01)$ at $Q^{2}$ values in the perturbative region $\left(Q^{2}\right.$ of a few $\left.\mathrm{GeV}^{2}\right)$ is still completely unknown. Measurement of the structure function $F_{2}$ and of the longitudinal structure function $F_{L}$ would give access to the gluon distribution in nuclei at very low $x$. In Figure 9 , the ratio of gluon distributions extracted from the longitudinal structure function is shown for 10 nucleon $f b^{-1}$ data for DIS and lead nuclei. Only statistical errors are shown. It would provide an impressive discrimination between the various models of shadowing in nuclei. Only statistical errors are shown The effects are so large that systematics should not spoil the physics message in the low $x$ regime which is relevant for formation of hot and dense gluonic matter when nuclei are smashed together at RHIC and the future LHC. 


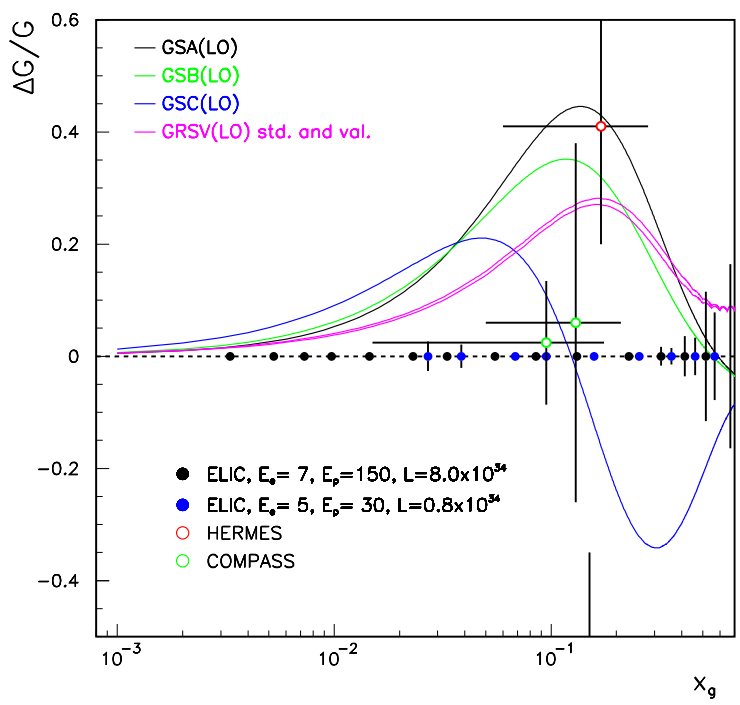

Figure 8: Projected uncertainties in $\Delta G(x) / G(x)$ from the charm production at the EIC. The integrated luminosity is $10 \mathrm{fb}^{-1}$ for the $10 \mathrm{GeV}$ electron on $250 \mathrm{GeV}$ proton measurement, and $2.5 \mathrm{fb}^{-1}$ for $5 \mathrm{GeV}$ electrons on $50 \mathrm{GeV}$ protons. For comparison, COMPASS and HERMES data points are shown.

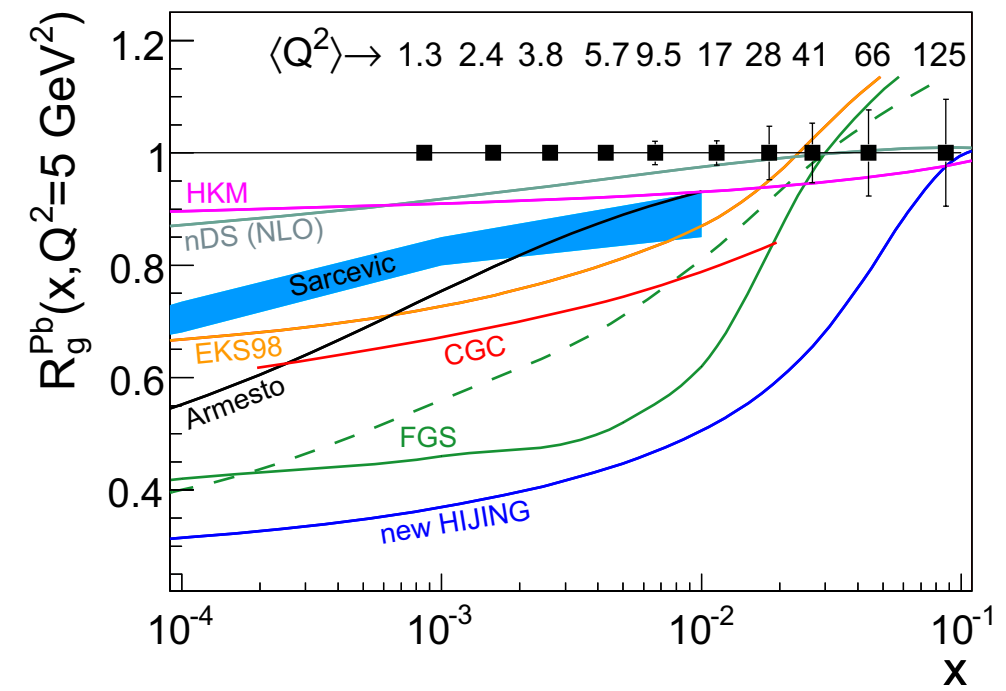

Figure 9: The ratio of gluon distribution in $\mathrm{Pb}$ nuclei to those in deuterium. The error bars correspond to statistical uncertainties for $10 / A \mathrm{fb}^{-1}$ at the EIC. A variety of model predictions which differ widely are shown. 


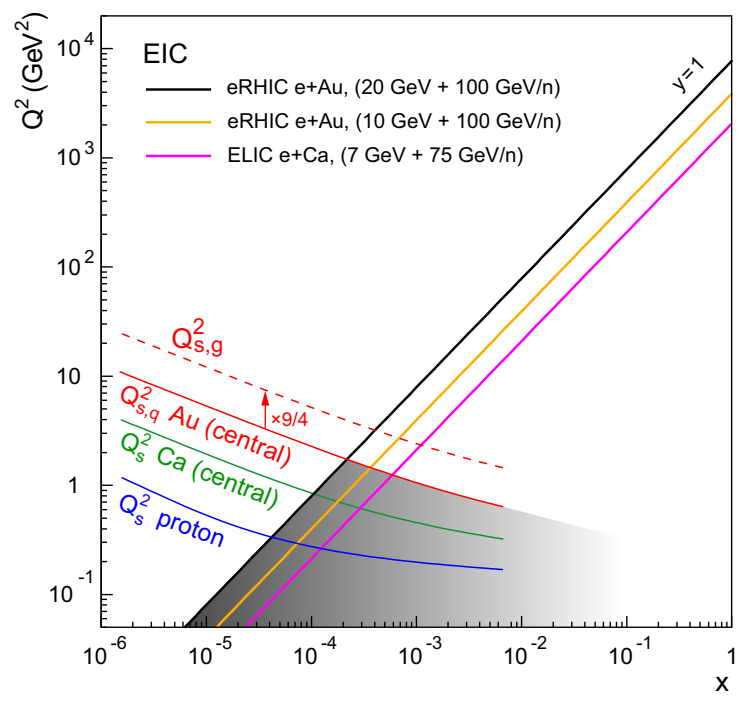

Figure 10: Kinematical acceptance in the $\left(Q^{2}, x\right)$ plane for the two concepts to realize EIC, eRHIC and ELIC. Lines showing the quark $Q_{s, q}^{2}$ or the gluon $Q_{s, g}^{2}$ saturation scale are superimposed.

$Q C D$ at low $x$ The strong rise of the gluon distribution as $x \rightarrow 0$ observed at HERA suggests that the gluon density in the proton attained its maximum values and saturates at lower $x$ than accessed at HERA in the proton, or at even a bit larger $x$ values in heavy nuclei. The saturation value $Q_{s}^{2}$ on gold could be around $2 \mathrm{GeV}^{2}$ at $x=10^{-3}$, a domain fully accessed at EIC ( Figure 10).

\section{$3.3 \quad$ LHec}

The LHC will explore a new range of mass and energy which goes far beyond the domain of HERA ( $~ 300 \mathrm{GeV}$ center-of-mass energy). An attractive proposition for an e-p collider [20] operating in the energy domain of the LHC is to make use of the $7 \mathrm{TeV}$ LHC $p$ beam by colliding it with an intense electron and positron beam stored in a ring mounted above the LHC, a Large Hadron Electron Collider (LHeC). There appears to be sufficient space to place the lepton beam line above the LHC magnets in the arc sections. A feasibility study using an electron ring of $70 \mathrm{GeV}$ energy leads to an estimated luminosity of about $10^{33} / \mathrm{cm}^{2} / \mathrm{s}$, at a center-of-mass energy of $1.4 \mathrm{TeV}$ (Figure 4). It is premature to have a time schedule. However a new ring cannot be installed before the LHC has produced physics and has, very likely, been upgraded to higher luminosity.

\subsubsection{Highlights of the Physics at LHeC}

The physics potential would not only be to increase by more than one order of magnitude the $x$ and $Q^{2}$ limits reached at HERA in e-p collisions but also, with heavy ions in the LHC ring, to study the e-A interactions in a completely unknown domain. Today the most attractive physics motivation is probably the $1.4 \mathrm{TeV}$ energy in the center-of-mass of the 


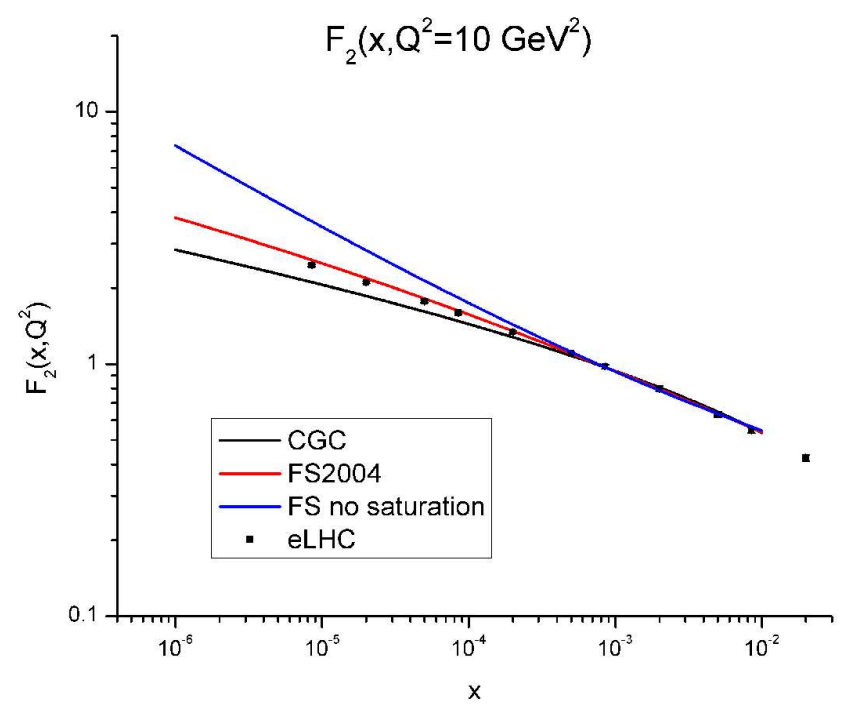

Figure 11: Predicted uncertainty on $F_{2}$ at very low $x$ with the LHeC. The integrated luminosity is $1 \mathrm{fb}^{-} 1$. The statistical precision is below $0.1 \%$ and systematics are about $1-3 \%$.

electron-parton interaction, a very promising domain for new physics. Workshops for a deeper evaluation of the physics potential and a thorough evalation of the LHeC physics in its relation to the LHC will be organised [21]. A few examples can already give a foretaste of the physics potential thanks to the unprecedented very high luminosity and the very high energy.

Very very low $x$ physics HERA has taught us a lot on low $x$ physics but many questions are not fully answered. Figure 11 shows how precise data at very low $x$ could clearly establish saturation at $Q^{2}$ values where perturbative QCD calculations apply [22]. It could distinguish between models of saturation.

Precision $Q C D$ The gluon density extracted from the QCD fit of the inclusive cross section at HERA has still an overall error of about $20 \%$ at $Q^{2}=4 \mathrm{GeV}^{2}$. Simulation shows (Figure 12) that the experimental precision of the gluon density at LHeC could be of about $3 \%$ at low $x$, down to $x=10^{-6}$ [23]. It should also be also possible to extract from the scaling violation of the structure function $F_{2}$ the strong coupling constant $\alpha_{s}$ with a relative precision of three per mil at the $Z$ mass [23].

Low $x$ in protons and nuclei In eA interactions $\mathrm{LHeC}$ extends by three orders of magnitude towards lower $x$ the range so far reached in fixed target experiments. In symbiosis with RHIC and ALICE it could help disentangling Quark Gluon Pasma from shadowing or parton saturation effects. In Figure 13 the gluon density has been extrapolated from HERA measurements towards lower $x$. It shows how the saturation point (also called unitarity limit) could be reached in e-p collisions at $Q_{s}^{2} \sim 5 \mathrm{GeV}^{2}$ and at much higher $Q_{s}^{2}$ values in e-A interactions when the $A^{1 / 3}$ increase of the gluon density in nuclei is taken into ac- 


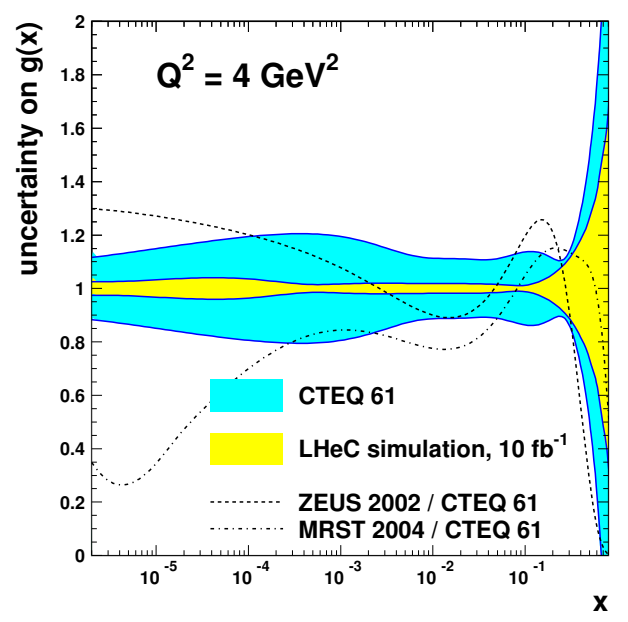

Figure 12: Expected uncertainty on $g(x)$ at the LHeC.

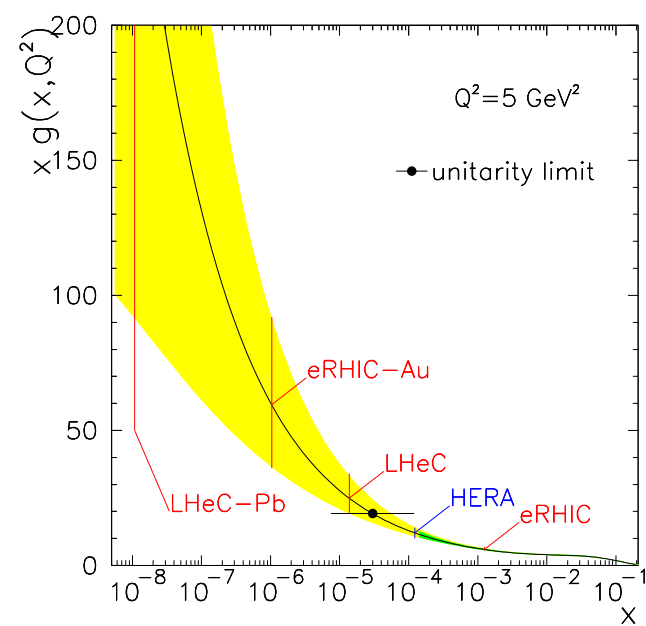

Figure 13: The gluon distribution from a NLO DGLAP QCD analysis of H1 data extrapolated to much lower values of $x$. The unitarity limit together with the region which can be accessed in e-p, e- $\mathrm{Au}$ and e-Pb scattering at the EIC and the LHeC are shown. 


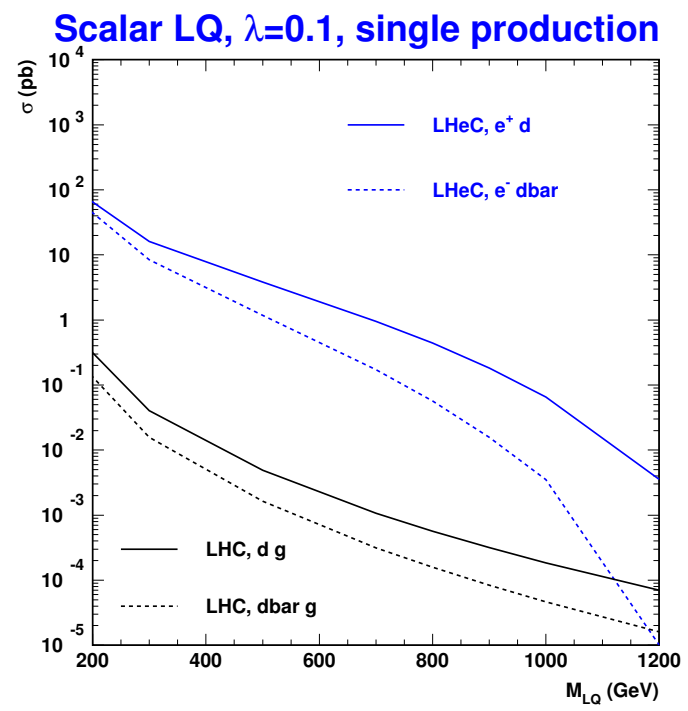

Figure 14: Single LQ production cross section at the LHeC (top) and LHC (bottom) for a scalar LQ coupling to $e^{+} d$ with a coupling constant $\lambda=0.1$. The integrated luminosity is $100 \mathrm{fb}^{-1}$ for the LHC and $10 \mathrm{fb}^{-1}$ for the LHeC.

count [22]. The saturation point is likely to be observed in e-p scattering at the LHeC and its effects in e-A scattering should be very large.

Physics Beyond the Standard Model Sensitivity to unknown physics beyond the Standard Model can be quantified within model assumptions. The Leptoquark production is an example. The high energy of the $\mathrm{LHeC}$ extends the mass range of single Leptoquark production up to $\sim 1 \mathrm{TeV}$ [24], the same limits as in the pair production at LHC. An e-p collider, providing both baryonic and leptonic quantum numbers in the initial state, is unique to study properties of an electron-quark resonance. A measurement of the asymmetry between the $e^{+} p$ and $e^{-} p$ cross section would determine the fermion number of the Leptoquark production [24]. The single Leptoquark production at the LHC could as well provide the Fermion number by comparing the signal cross sections with an $e^{+}$and an $e^{-}$coming from the decay of the Leptoquark. However, the single Leptoquark production cross section at LHC is two orders of magnitude lower than at the LHeC ( Figure 14).

\section{Complementarity of lepton-hadron and hadron-hadron facilities}

We have seen in the example of the leptoquark how the LHeC would provide complementary information to the LHC : discovery at LHC and measurement of quantum numbers at LHeC. 
More generally, the physics adressed in DIS facilities provides complementary information to hadron hadron collision or can be complemented by information from hadron hadron collisions, in particular in PDFs determinations, study of nuclear matter or spin structure of the nucleon.

\subsection{Complementarity of p-p and e-p on PDFs}

When extracting the PDFs, the main source of informations on quark densities comes from DIS experiments on fixed target at HERA. Complementary information has been provided by Drell Yan pair, prompt photons and di-jets in p-p scattering. At this workshop it has been shown [25] how measurements from LHC may improve knowledge of gluon density at low $x$ from the $\mathrm{W}$ rapidity distribution and at high $x$ from the high transverse momentum jets. The $\mathrm{W}$ asymmetry should provide an additional constraint on low $x$ quark distributions. However, the present contribution of the Tevatron to PDFs shows that we should not expect miracles. It is not a substitute to lepton-nucleon DIS.

\subsection{Complementarity of polarized $\vec{e}-\vec{N}$ and $\vec{p}-\vec{p}$ facilities}

So far information on spin structure of the nucleon comes from polarised lepton-nucleon fixed target experiments at SLAC, CERN (muon beams) and DESY down to $x \sim 0.01$. Further data on the densities $\Delta u, \Delta \bar{u}, \Delta d, \Delta \bar{d}$ at relatively large $x$ in the nucleon will come from RHIC through its W-physics program, and from the $12 \mathrm{GeV}$ upgrade at JLAB. Only an e-p collider, as the EIC, with polarised electron and protons (deuterons) can give access to the low $x$ domain which is crucial for the determination of the integral of spin distributions and to test the very fundamental Bjorken Sum Rule [26].

\subsection{Complementarities of $\mathrm{p}-\mathrm{A}$ and $\mathrm{e}-\mathrm{A}$ studies}

The complementarity between lepton-nucleus, proton-nucleus and nucleus nucleus studies can be shown in two different types of study of nuclear matter :

Saturation at low $x$ Saturation models predict that the saturation limit will be well inside the $\left(Q^{2}, x\right)$ range probed at RHIC and LHC in $p A$ collisions [27]. However factorization is uncertain in the strong gluon field regime even for inclusive observables [28]. It may turn out that e-A data in the same $(Q 2, x)$ region are vital to understand the dynamics of saturation.

Hot and dense matter To further explore and quantify the properties of the new collective behaviour observed at RHIC in ion-ion collisions, upgrades at RHIC are planned. At the LHC, p-p, p-A and A-A collisions will provide substantially higher energies. To fully understand the dynamics of a Quark Gluon Plasma, a precise knowledge of the initial parton distribution in nuclei, would be an important asset which can be independently extracted from e-A collisions in the same kinematic range at the EIC [29].

\section{Conclusion}

To conclude I would like to express a few personnal comments:

- The physics of DIS will not stop with the end of data taking at HERA. 
- The final data of HERA will be an important asset to high energy physics. Precision of most results is still dominated by experimental and theoretical systematic uncertainties. All efforts should be made to achieve the highest possible precision. It will be a safe and cheap investment in the future.

- The $12 \mathrm{GeV}$ upgrade of the electron beam at Jefferson Lab is on the right track to be finally approved and to get new and precise insights into the valence quark region at $Q^{2}$ of a few $\mathrm{GeV}^{2}$.

- The proposed Electron Ion Collider (EIC) at Brookhaven or Jefferson lab would be a real break through in nucleon spin and nuclear matter physics. It's urgent to establish a process to choose the best design. It should be a trade-off between luminosity, energy, time schedule and cost.

- The project of a Large Hadron Electron Collider is a very attractive complement to the proton-proton and ion programmes at CERN. But in Europe it would be in competition to all other future facilities beyond the fist phase of the LHC. Today it is quite uncertain to be supported by the high energy physics community and the european funding agencies, unless new developments at LHC indicate the physics case becomes even more desirable.

\section{Acknowledgments}

I thank all the speakers of the Future of DIS session for their enthusiastic talks, and Abbay Deshpande, Max Klein, Naomi Makins and Alexei Petrukhin for valuable help.

\section{References}

[1] Slides: http://indico. cern. ch/contributionDisplay . py? contribId $=17 \&$ sessionId $=2 \&$ conf $I d=9499$

[2] R. Hofstadter, Phys. Rev. 98217 (1955).

[3] X. Ji, Phys. Rev. Lett. 78610 (1997).

[4] JJ. Aubert et al. EMC Collaboration, Phys. Lett. B123 275 (1983).

[5] A. Deshpande et al., Ann. Rev. Nucl. Part. Sci. 55165 (2005).

[6] M. Arneodo, Phys. Rep. 240301 (1994).

[7] L.V. Gribov, E.M. Levin and. M.G. Ryskin, Phys. Rep. 1001 (1983).

[8] H. Kowalski, T. Lappi and R. Venugopalan, Nuclear enhancements of universal dynamics of high parton densities, arXiv:hep-ph/07053047 (2007).

[9] N. Armesto, J. Phys. G32 R367 (2006).

[10] EIC Collaboration, Physics Opportunities with $e+A$ Collisions at an Electron Ion Collider, White paper final, April 4 (2007).

[11] I. Abt et al. H1 Collaboration, Nucl. Phys. B407 515 (1993); M. Derricket al. ZEUS Collaboration, Phys. Lett. B316 412 (1993).

[12] G. Altarelli and G. Parisi, Nucl. Phys. 126298 (1977);

Y.L. Dokshitzer, Sov. Phys. JETP 46641 (1977);

L.N. Lipatov, Yad. Fiz. 20181 (1974);

V. Gribov and L. Lipatov, Sov. J. Nucl. Phys. 15438 (1972).

[13] M. Derricket al. ZEUS Collaboration, Phys. Lett. B315 481 (1993); T. Ahmedet al. H1 Collaboration, Nucl. Phys. B429 477 (1994).

[14] W.K. Tung, Update on CTEQ global analyses, these proceedings.

[15] S. Ferrag, arXiv:hep-ph/0407303 (2004), proceedings of 39th Rencontres de Moriond on QCD and High-Energy Hadronic Interactions, La Thuile, Italy (2004). 
[16] A. Lung, Future plans at Jefferson Lab: 12 GeV Upgrade and ELIC, these proceedings.

[17] EIC Collaboration, A High Luminosity, High Energy Electron-Ion-Collider, White paper final, April 24 (2007).

[18] S. Aronson, Future plans at BNL: RHIC-II and eRHIC, these proceedings.

[19] A. Bruell, $\Delta G$, spin/flavour and GPDs at EIC, these proceedings.

[20] J.B. Dainton et al., Deep Inelastic Electron-Nucleon Scattering at the LHC, arXiv:hep-ex/0603016 (2006).

[21] J.B. Dainton, $\mathrm{LHeC}$, these proceedings.

[22] P. Newman, Low x physics at the $\mathrm{LHeC}$, these proceedings.

[23] M. Klein, Parton Distributions from the $\mathrm{LHeC}$, these proceedings.

[24] E. Perez, LHeC and Physics Beyond the Standard Model, these proceedings.

[25] A.M. Cooper-Sarkar, Impacts and constraints on PDFS at ATLAS, these proceedings.

[26] J.D. Bjorken, Phys. Rev. 1481467 (1966); Phys. Rev. D1 1376 (1970).

[27] D Kharzeev and M. Nardi, Phys. Lett. B 507121 (2001);

D Kharzeev, E. Levin and M. Nardi, Nucl. Phys.A 747609 (2005).

[28] J.W. Qiu and G. Sterman, Nucl. Phys.B353 137 (1990).

[29] A. Accardi et al., in CERN Yellow report, Hard Probes in Heavy Ion collisions at the LHC, arXiv:hep$\mathrm{ph} / 0308248$ (2003). 